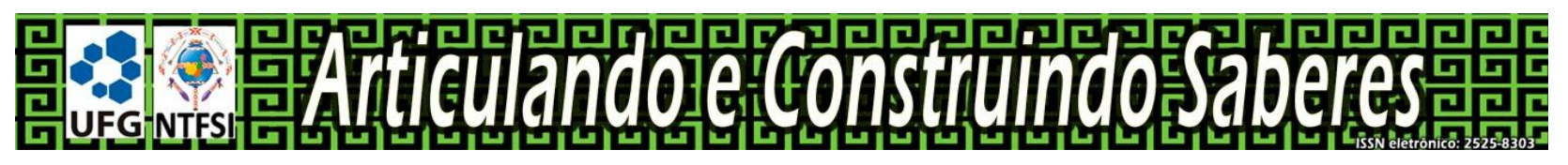

DOI:10.5216/racs.v5.65253

\title{
Antagonismo territorial, una tensión productiva para pensar el colonialis- mo chileno
}

\section{Sergio Caniuqueo Huircapan ${ }^{1}$}

\section{RESUMEN}

El presente artículo intenta hacer una síntesis de dos artículos publicados con 15 años de diferencia, sobre un tema tan central como son las percepciones territoriales antagónicas, en procesos de luchas y reivindicaciones de territorios indígenas, que para este caso se trata del Pueblo mapuche ubicado en Gulu Mapu, cuyo telón de fondo es el colonialismo chileno. El enfrentamiento, en términos de concepciones distintas, tiene efectos en distintos ámbitos, pero al reconocerlas también abren la posibilidad de pensar situaciones de convivencia territorial.

PALABRAS ClAVE: Antagonismo Territorial. Colonialismo. Mapuche.

\section{Antagonismo territorial, tensão produtiva para pensar o colonialismo chileno}

\section{RESUMO}

Este artigo tenta fazer uma síntese de dois artigos publicados com 15 anos de intervalo, sobre um tema tão central quanto as percepções territoriais antagónicas, em processos de lutas e reivindicações de territórios indígenas que, neste caso, é sobre o povo Mapuche, localizado em Gulu Mapu, cujo pano de fundo é o colonialismo chileno. O confronto, em termos de diferentes concepções, tem efeitos em diferentes áreas, mas ao reconhecê-lo abre também a possibilidade de pensar situações de convivência territorial.

PALAVRAS-CHAVE: Antagonismo Territorial. Colonialismo. Mapuche.

\footnotetext{
${ }^{1}$ Unversidade do Chile, Santiago, Chile. E-mail: sergiocaniuqueo@gmail.com.
}

Dossiê Redes de Inter-aprendizaje: nuevas cartografías interculturales y algunas propuestas de transformación 


\section{Introducción}

Hace 30 años apareció en los discursos del movimiento mapuche, en el sector de Gulu $\mathrm{Mapu}^{2}$, el concepto de territorio, incluso en tensión con organizaciones chilenas, que a ese tiempo ya habían desarrollado prácticas paternalistas frente a los mapuche, como ocurre con el reclamo público que hace la organización "FUTATRAWUN KINEWAN MAPUCHE" Coordinadora Unitaria Mapuche a Renata Zamorano, Presidente de la Comisión de Derechos Humanos, IX Región:

1.-Ante el hecho consumado de que ustedes como Comisión de Derechos Humanos, están llevando a cabo un Seminario sobre Autonomía, Soberanía y Territorio para los pueblos étnicos, ratificamos nuestra declaración pública que hicimos el pasado mes de marzo, donde señalamos que todas las propuestas que conciernen a los pueblos étnicos, sean políticos, económicos, sociales, culturales, etc.; corresponde asumirlos a los propios pueblos, mediante sus organizaciones representativas (1988, p. 7).

Sin duda, esto había sido parte de un proceso en la cual las organizaciones mapuche buscaban construir un proyecto político propio, en el marco de la dictadura militar que se había instaurado en 1973 con la instalación de Junta Militar en Chile, encabezada por Augusto Pinochet. Además, el discurso de territorio tenía su correlato en el proceso de subdivisión de las comunidades, que hasta ese entonces estaban bajo un régimen de propiedad colectiva. A partir del Decreto 2568, al subdividir las comunidades se entrega el título de propiedad privada a las familias, pero al mismo tiempo se decretaba que se suprimía la calidad indígena de la tierra y de sus ocupantes. A ese tiempo las organizaciones mapuche veían un atentado a la continuidad de pueblo y un nuevo despojo a sus tierras, de hecho gran parte de los esfuerzos de los mapuche organizados fue frenar a la venta de tierra y arriendos a 99 años que consideraba el decreto, para ello realizaron diversas alianzas con organizaciones de la sociedad civil chilena, principalmente la iglesia católica.

En el 2005, publique un artículo llamado Antagonismo en las Percepciones Territoriales, Un Marco de Interpretación (2005), en la cual me planteaba ver como se construían las percepciones sobre el territorio, y porque en el caso de la sociedad chilena y mapuche se daba

2 Cuando hablamos de Gulu Mapu, nos referimos al territorio del Pueblo Mapuche que se ubica actualmente bajo el Estado de Chile, mientras que Puel Mapu, se refiere al territorio que se ubica bajo el Estado Argentino.

Dossiê Redes de Inter-aprendizaje: nuevas cartografías interculturales y algunas propuestas de transformación

R. Articul.const.saber, 2020, v.5: e65253 
una visión antagónica en el uso y en la significación, a continuación, tomo gran parte de ese artículo para reposicionar la discusión que comencé hace más de 15 años.

\section{Kimun Mapuche una perspectiva de conocimiento}

Quienes comienzan a estudiar al mundo mapuche, una de sus primeras observaciones se centra en los conceptos que componen la palabra mapuche, las traducciones rápidas hacen que el concepto Mapu sea interpretado como "tierra", y Che como "gente", así llegan a la conclusión que Mapu-Che sería “gente de la tierra”. Esta concepción es errónea para entender la dinámica del pueblo mapuche y su lógica espacial. El concepto Mapuche tiene una dimensión ontológica, sustentado en el Mapu (espacio o dimensión) y el Che (sujeto socializado), investigadores como Guillaume Boccarra (1999) han situado el concepto mapuche a mediados del siglo XVII, con lo cual se le ha dotado de historicidad al concepto. El Che como concepto ha sido conocido desde el primer contacto con el winka (extranjero). Este autor señala que la primera identificación frente al español fue la de Reche, o sea, Re (origen) y Che (persona) lo que daría como sujeto originario. Siguiendo a Boccara, el concepto mapuche sería parte una etnogénesis, a partir de la tensión bélica entre hispanos y mapuche, en la cual hay una necesidad de diferenciarse de una manera más taxativamente del invasor al autodenominarse Mapu-Che, él "ser humano" de este territorio, en tal sentido, se van estructurando los límites étnicos a partir de elementos físicos. En otras palabras ya no es suficiente identificarse como sujeto originario, sino que había que enfatizar en el territorio esa diferencia, en el Mapu, que a la vez hace alusión al espacio físico, simbólico y espiritual de los sujetos y que en cada uno de estos ámbitos hay una diferencia total frente al winka o no mapuche.

Para seguir en nuestra argumentación nos centraremos en una epistemología mapuche, esto quiere decir que utilizaremos los conceptos y categorías desde el mapuzungun, estableciendo como eje lo que podría articular a la sociedad y percepción territorial, estableciendo algunos límites desde la temporalidad y la territorialidad. Los conceptos Mapu y Che, han existido siempre y son los que nos permiten tener: una visión de mundo y la relación con lo que nos rodea en la dimensión física, social, simbólica y espiritual.

El Che es la persona, pero como agente socializado, alguien que está dentro de un proceso y cumple ciertos criterios fijados por la sociedad, es decir que así como se puede ser persona se

Dossiê Redes de Inter-aprendizaje: nuevas cartografías interculturales y algunas propuestas de transformación 
puede dejar de serlo (QUIDEL \& JINEO, 1999, p. 150). El Che depende de la formación de valores, por lo cual hay una construcción axiomática, la cual responde a la ética, lógica y razonamiento mapuche. Cada Che es un elemento particular que se establece a partir de una serie de elementos exógenos y endógenos, la potencialidad o el capital cultural (en el sentido bourdiano) del Che, en elementos como el tüwun (lugar de origen) y su küpalme (tronco familiar) se funda la identidad dentro del Wallmapuche (AUKIÑ WALLMAPU NGULAM, 1997, p. 99). En este punto hay que plantear algunas consideraciones, que el tüwun y el kükam, también hacían diferencia en términos segmentarios al interior de la sociedad mapuche, no existe la idea de que todos los sujetos son iguales o están en el mismo plano. Esto es importante señalarlo, ya que esto ha llevado a pensar en una imagen idílica de la sociedad mapuche, en la cual todos los sujetos son iguales o existe una vida comunitaria sin conflicto. Por ejemplo, Manuel Mañkelef o Maquilef (castellanizado) planteaba en su libro "Las Tierras de Arauco", a principios del siglo XX, se oponía a una ley indígena que dejaba en un mismo plano a un Longko (al jefe de un linaje) y a un Kona (un subordinado de un longko), tenemos casos a fines del XIX y principios del XX, donde konas tomaron el apellido del longko al cual eran leales y utilizaron el diminutivo de pichi (literalmente se traduce como pequeño) en este sentido por ejemplo, los Pichineculman serían kona de los Neculman, en el caso de una investigación en Boroa (CANIUQUEO, 2012), si bien los descendientes de los Pichineculman reconocían relación con los Neculman, es tenía relación con que ellos actuaban como weupife (oradores o tribunos) con lo cual representaban el pensamiento del territorio frente a otros longkos provenientes de otros territorios, a los Neculman se les reconocía su habilidad para negociar con los wingka chilenos, pero su debilidad estaba en su kimun (sabiduría) y rakizuam (pensamiento) frente al mundo mapuche, es por ello, que los Pichineculman cubrían esta necesidad, esto último nos muestra una complejidad de la subalternancia, hoy por ejemplo, se traduce la palabra Kona como trabajador, cuando en el siglo XIX, podía ser la de un colaborador, en el ámbito intelectual como en el trabajo físico, o en la guerra, como podía ser un weychafe (guerrero). El caso, es que no hay una sociedad igualitaria y el tuwün y el Küpan, cumple con la función de marcar esa diferencia y establecer el papel del linaje de acuerdo a su territorio. Hoy, hay muchas personas mapuche que utilizan estos conceptos sin esta carga, sino como un conceptos que permita la inclusión de sujetos ligados a un mismo

Dossiê Redes de Inter-aprendizaje: nuevas cartografías interculturales y algunas propuestas de transformación 
territorio, dando a la identidad un sentido más amplio y menos segmentario que lo que tenían en el siglo XIX hasta parte de la segunda mitad del siglo XX.

Ligado al concepto de tüwun se desprenden dos conceptos que se relacionan con los Che pertenecientes al Lof, los amunche, familias que han existido desde tiempos inmemoriales y los akunche, como aquellos que han venido de otros mapu y se asienten en este territorio (QUIDEL \& CANIULLAN, 2002, p. 4), esto permite analizar el tema de los linajes o los rayñma los cuales tienen relación con el concepto de kupalme que anteriormente señalábamos. Podemos agregar que, de estas relaciones entre diferencias y semejanzas que tenían los Che para convivir en un mismo espacio, dependían de su az mapu y su ixo fij mogen, esto se hacía en un weupitun donde se veían si las características que traían los akunche coincidían o permitirían una armonía con los amunche, de coincidir se aumentaría o ampliarían los conocimientos, alianzas territoriales, semejanzas en los gijatun, concurrencia a trawun, koyaltun o gulamtrawun de rewe y ayjarewe. Pero también existía la posibilidad de que los akunche y amunche no poseyeran el mismo az mapu y con ello se establecía las explicaciones de los conflicto entre comunidades, esto conflictos permitían que las redes de colaboración se extendieran territorialmente, y en caso de conflictos, las redes de cada grupo se vieran enfrentadas y generan colaboraciones en el caso de situaciones violentas, con ello queremos mostrar que no existe una cosmovisión mapuche que hermane a todos los sujetos, existen nociones que son compartidas, pero también las particularidades nos pueden llevar a los conflictos, sin embargo también existían fórmulas para evitar enfrentamientos o en el caso de existir pudieran existir negociaciones de paz, aquí destacamos la presencia del koyagtun. Los conceptos de Akunche y Amunche, identificaban también con quienes emparentarse, y establecer quien ejercerse los liderazgo en un territorio, en nuestras observaciones en terreno, por ejemplo pudimos observar como en el sector de Ragiñtuleufu, el liderazgo con los años paso a amunche a los akunche, los apellidos con terminación Queo dejaron de ejercer el liderazgo tradicional, ante ellos los apellidos con terminación Laf asumieron este liderazgo, pero ello ha generado una serie de tensiones en el territorio, lo cual revela que los cambios de liderazgo viene con una tensión, el paso del tiempo puede llevar a tres caminos, a una aceptación de los nuevos liderazgos (a partir de un declive de las tensiones en el largo plazo) a un enfrentamiento de liderazgos permanente o a una desarticulación y fragmentación del territorio, en la cual cada liderazgo agrupe a un sector.

Dossiê Redes de Inter-aprendizaje: nuevas cartografías interculturales y algunas propuestas de transformación 
Hay personas que aparecen como reche (personas originales) o kumeche (las cuales sirven de modelos a seguir en términos positivos) también existe el wezache (alguien que no tiene buen entendimiento) o Ka keche (otras personas) estas últimas establecen que no tienen sin ninguna relación y/o compromiso, solo existen con vinculaciones a grupos que se desconocen, esta palabra igual puede ser despectiva, ya que el no reconocimiento de ningún vínculo puede cuestionar el considerarlo como un ser humano valido o confiable. Son modelos que existen, cuya condición y estatus están referenciados en su capacidad de responder a ciertos valores que, a partir de las formas de transmisión de conocimiento, se buscan mantener al interior del lofche, como lo plantea Domingo Carilao (Mariman, 2000, p. 3) quien también afirma que "Las sociedades indígenas socializan a sus miembros en un contexto dado, a través de sus valores, creencias, costumbres y normas propias que éstas definen. En efecto, la sociedad mapuche, en tanto sociedad indígena, poseerá también estos mecanismos para llevar a cabo este proceso" (CARILAO, et al., 1998).

En un informe de sistematización que realizamos en el 2010, el consejo Azeluwam establecía que el sujeto socializado que podía sostener a la sociedad mapuche, en términos de conocimientos era quien desarrollaba el Che Geal, el cual es un sujeto que logra autosustentarse en el mundo y dar sustento al mundo en el que esta inserto:

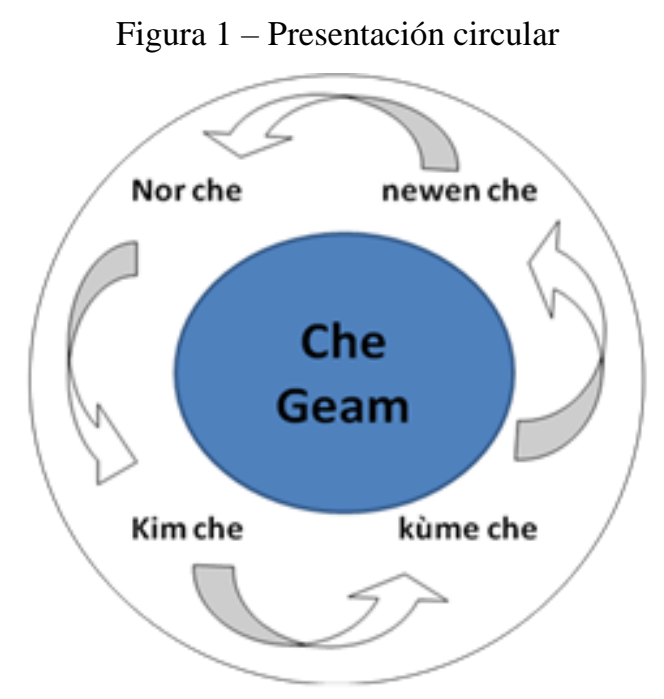

Elaboración: Equipo técnico Consejo de Educación Mapuche Azeluwam 
La presentación circular permite dar cuenta de un proceso múltiple, complejo y dialéctico en la conformación del Che, a partir de una retroalimentación integral en todos sus aspectos, los que a su vez se encuentra normado por los aspectos culturales mapuche.

Norche: quien reúne condiciones físicas, psicológicas y espirituales que se consideran desde el normogen, su práctica cotidiana que se caracteriza por su relación equilibrada con su entorno social, natural y sobrenatural.

Kimche: quien ha desarrollado conocimientos, sus competencias para aprender y enseñar distintos tipos de conocimientos y saberes, así también posee una coherencia con los valores aprendidos y es capaz de demostrarlo a partir de sus actos e ideas.

Kumeche: quien se ha iniciado o abierto a la espiritualidad, solidaridad, capacidad de ser comprensivo y pensamiento crítico, para que pueda tener conocimientos y bienes materiales, con la conciencia de que sus logros son productos de sus buenos actos en el aspecto social, espiritual y natural que constituyen el lof.

Newenche: quien constituye todos los aspectos que componen el ser norche, kimche y kümeche, desarrollándose en él su kupalme y su coherencia con su tuwun y kupan.

Cuando hablamos de norche nos referimos al aspecto más básico que puede desarrollar una persona, como veremos más adelante, es la condición física, psicológica y espiritual que lo hace ser aceptable ante los demás, pero no es una condición intrínseca, sino que esta daba por su nacimiento como una persona sana y eso indica que su familia no tuvo ninguna transgresión, es trabajadora porque su familia la oriento, es respetuoso también por lo mismo y participa de la comunidad porque su familia lo hace, pero al mismo tiempo aprende de la comunidad y eso le da su identidad también, es aquí donde comienza articular su identidad individual y la de su comunidad. El kimche es alguien que fuera de ser norche comienza a mostrar cariño y admiración por el conocimiento, afloran sus habilidades para obtenerlo y desarrollarlo, uno de los aspectos clave es su comunicación y su conversación, ello es lo que lo va resaltando su condición pues no es solo conversar, es saber llevar un tema y desarrollarlo, pero también involucra el saber escuchar y reflexionar. Desde esta perspectiva el kumeche no solo significa quien vive bien, su valoración se encuentra en cómo se llega a ser, y el vivir bien no se traduce en quien tienes bienes materiales sino su indicador se encuentra en las relaciones que mantiene con todo lo que lo rodea,

Dossiê Redes de Inter-aprendizaje: nuevas cartografías interculturales y algunas propuestas de transformación 
desde la familia, la comunidad, los seres vivos y la naturaleza, así como sus intenciones de ayudar a la comunidad. Por último, el newenche quien se desarrolla en todas sus dimensiones biológica, social, psicológica y espiritualmente, cumple con su kupalme, con su rol social, entiende al igual que el kumeche que lo que tiene o ha logrado obtener no es por él sino por todo lo que lo que está rodeando desde su familia, la comunidad, la naturaleza, y la dimensión espiritual. De ahí que el ideal de estar personas sean serviciales, humildes, empáticos, solidarios y que piensen siempre en el bienestar colectivo antes que en el de ellos. (CANIUQUEO, 2010, p. 20-21)

Estos valores entran en en momentos cruciales como un gulamtrawun, reunión donde concurren longko y gulamtuchefe (personas que tiene buenos consejos o la suficiente claridad para orientar), donde el objetivo final es tomar las resoluciones más asertivas frente a conflictos y perspectivas futuras de los lofche, en un contexto político. Con esto queremos decir que todo che en la medida que cultiva los valores antes mencionados va estructurando una división al interior del lofche, la cual está dada por el proceso de "ser" persona.

Cada persona cultiva un saber y características especiales, que están dadas por su relación con su mapu (espacio) y las dimensiones que éstas tienen, como también en los elementos que permiten dicha relación. En la interpretación mapuche podríamos decir que el che es un medio que interrelaciona los elementos constituyentes de su mapu, es quien debe mantener el equilibrio, pero, son los elementos que posee el mapu los establecen su característica, su az mapu, la identidad territorial, debido a esto Quidel y Jineo plantean que es necesario apreciar que el che se constituye a partir de lo que lo rodea y que está en igual condición en el nag mapu con el ko (agua), lafken (mar o lago), etc (1999, p. 153).

Al hablar de mapu es erróneo pensar en tierra (püji) que es el recurso material que nos sostiene a nosotros y todo lo que nos rodea (p. 149), el concepto se relaciona con espacio, en su dimensión física, social y simbólica. Nuestro conocimiento se estructura a partir de cómo nos relacionamos con nuestro espacio y sus dimensiones. Por ello, el concepto Mapu “... es una construcción que la sociedad mapuche ha realizado para fundamentar su existencia y estructuración...” (p. 148), siendo el Waj Mapu, el concepto englobador de los mapu, universo tangible e intangible donde viven todos los seres.

Dossiê Redes de Inter-aprendizaje: nuevas cartografías interculturales y algunas propuestas de transformación R. Articul.const.saber, 2020, v.5: e65253 
Para plantear nuestro cosmos hay que aclarar dos conceptos, uno, el Waj mapu como una estructura circular horizontal que une a todas los espacios y, dos, está el concepto del Wojontu mapu, el cual se caracteriza por la unión de todos los mapu de forma vertical (CHILKOWE, 2001, p. 15), explican además la redondez de la tierra, la concepción de universo y de interrelación existentes en los mapu entendidos como espacios y dimensiones (JINEO \& QUIDEL, 1999, p. 149). Se aclara que al Waj mapu lo relacionamos con el universo material e inmaterial (QUIDEL, p. 2002) mientras el Wojontu se puede relacionar con lo que nos rodea.

Por lo general, se habla aproximadamente de siete dimensiones de mapu, partiendo de arriba hacia abajo encontramos el Wente Wenu Mapu, lugar donde llegan los jejipun (oraciones y peticiones que son hecha de buena manera) y viven seres de otras dimensiones el Wenu Mapu, donde llegan algunas de las personas cuando mueren; Ragiñ Mapu; Anka Wenu Mapu; se encuentran los seres que protegen el Naq Mapu; Naq Mapu, es el espacio fisco donde habitan todos los seres de característica material e incluso inmaterial; Münche Mapu, que corresponde a todo lo que está bajo el subsuelo; y el zenchu mapu que es una línea vertical, desde arriba hacia abajo en el centro (CHILKOWE, 2001, p. 16). La funcionalidad de estos espacios se encuentran en rituales que sólo algunas autoridades mapuche dominan, esto se debe a que el pülli (espiritu) del che puede venir de uno de estos espacios lo mismo que su newen (fuerza), el che se constituye no solo de un espacio, por esta razón es difícil entregar una definición a cada concepto ya que depende del contexto en el cual está situado el che al interior del Waj mapu. A partir de estos mapu se va estructurando el conocimiento en términos de contenidos en el mundo mapuche.

Es importante, para entender el tema de territorialidad mapuche y la relación con otros mapu, el concepto de pülli, que se ha traducido como alma, de lo cual no estamos seguros que sea el concepto adecuado, es un elemento componente del che, y puede provenir de los distintos mapu que antes mencionamos, incluso Quidel (2002) habla de 12 mapu en total. El pülli tiene una fortaleza en los elementos físicos de que se encuentra en el Naq Mapu como los ríos, esteros, vertientes, así como del mapu del cual proviene. Por otro lado, el pülli circula al interior de un kupan, esto quiere decir de un linaje, es por ello, que mencionábamos que cada che pertenece a un kupan al interior de un lofche, cumpliendo una función especial, sea este de werken, longko, machi, etc, de esta forma se estructuran ciertas divisiones al interior del tejido social mapuche. Cada autoridad mapuche maneja un conocimiento específico de cada mapu, de ahí su kimun 
(sabiduría) y su newen (fuerza). Muchos cientistas sociales no han comprendido esta relación creando un imaginario del mundo mapuche, pasando por la encima de la pluralidad de las identidades territoriales, sus interrelaciones, en el mundo mapuche como en el mundo winka, y la actual forma en la cual el mapuche se relaciona con sus recursos.

Para entender las diferencias entre identidades territoriales mapuche hay que analizar históricamente la especificidad de sus pülli, es cierto que existen elementos transversales a todas las unidades territoriales como el gijatun, eltun (cementerio), trawun (reuniones), etc, pero la especificidad está marcada por cada identidad territorial. Añadiendo que la especificidad geográfica que a cada identidad le toca vivir y conocer ayuda a crear el az mapu de esa identidad, permitiéndole establecer elementos diferenciadores así como necesidades de articulación a otras unidades territoriales.

Para el caso de recibir un newen (poder) una autoridad tradicional, esta puede ser recibida por pewma (sueño), el poder no viene en forma unidireccional sino que depende del origen del pülli, por lo tanto, el pewma corresponde a un mecanismo en el cual se relaciona al che, que vive en el naq mapu, con su mapu de origen. A partir de ahí la persona va recibiendo la autoridad y el conocimiento para ejercer su función, esto se validad a partir de los pewma en personas del lofche $o$ en autoridades tradicionales que ratifican la ocurrido y señalan el tipo de comportamiento que debe adoptar el lofche.

Estos conocimientos son de carácter específicos, sólo tienen sentido en el mapuzungun, es por ello que son manejados por personas ligadas al kimun (sabiduría) y espiritualidad mapuche, las cuales se interrelacionan con los mapu e incluso viajando a ellos, esta información es reservada y personal, es por ello que las autoridades tradicionales hablan poco de ello, como señala el longko wenteche José Quidel, “... esto es lo que ha permitido que el pueblo mapuche se mantenga todavía, pero cada mapu nombrado son esferas específicas de conocimiento y las autoridades tradicionales mapuche sólo manejan algunas...” (QUIDEL, p. 2002). Es por ello que el concepto mapu es conocimiento e interrelación con el espacio, aprendiendo sus normativas que encierra lo físico, social y simbólico a través de sus prácticas ancestral.

En el caso del Naq mapu existe el Ixo Fij Mogen, lo cual equivale a las normas que establece el mapuche con su medio ambiente (Ibid; Identidades Lafkenche, Calasquenche, Nangche, Huilio, Makewe y Pewenche, 2002, p. 3), esto quiere decir que el conocimiento se

Dossiê Redes de Inter-aprendizaje: nuevas cartografías interculturales y algunas propuestas de transformación 
construye a partir de la relación con el medioambiente, estableciéndose normas que luego se transmiten. En todos los mapu existen reglas, lo que se establece con el concepto de Nor Mogen (reglas del lugar) y en la relación con las personas Az Mogen (reglas que caracterizan y diferencian) (AUKIÑ WALLMAPU NGULAM, p. 9-10), este cuerpo de normas caracteriza al lugar y permite establecer las reglas particulares, que lo diferencian establecen afinidad con otras entidades territoriales, esto es lo que se conoce con el concepto de Ad Mapu. Esto sumado al tuwün y su kupalme les da esa característica de Az, que permite relacionar a los che de un lof con las características que tienen un lugar, sean ambientales o geográficas, las cuales las han impreso en sus ceremonias o espacios simbólicos, o sea en la forma de hacer gijatun, eluwun, mafun, etc. De esta forma se estructura el derecho consuetudinario y costumbres dando paso al control del territorio.

Esto es la territorialidad mapuche, la interrelación, entre lo físico, lo social y lo simbólico, una estructura formada a partir de una base material que ha llegado a construir una super estructura que lo sustenta. Es necesario advertir que pese a las interrelaciones con los chilenos y los elementos introducidos, esto se mantiene en el pensamiento y la lógica mapuche de concebir la territorialidad. Las normas que sustentan a un longko gijatufe o una machi se basan en este modelo de sociedad, que no es un modelo cerrado, permitiendo introducir discursos y formas de entender la vida, manteniendo gran parte de este modelo.

El conocimiento mapuche se articula en espacios sociales o públicos, que son en el fondo espacios intrafamiliares, es ahí donde se manifiestan los conocimientos específicos que son manejados por cada tuwun y kupalme, donde cada familia cumple, o estaría en condiciones de asumir, cierta funcionalidad al interior del lofche, ya sea por su historia o porque hay elementos que los han caracterizado, esto es lo que posibilitan que presenten un rakizuam (pensamiento) como familia, en un trokinche o en un lof mapu, en el caso del az mapu este refleja el rakizuam de los lofche, es aquí donde se funda la posibilidad que se articulen en otras unidades como rewe y ayjarewe, las que quedan plasmadas en elementos rituales como el gijatun y trawuwe (lugares exclusivos de reuniones), como lo platea Dillenhay, toda sociedad deja plasmada su visión espacial y forma de entender la "realidad" es en los espacios simbólicos.

La dinámica espacial mapuche, antes de la imposición espacial del Estado Chileno era la siguiente: la unidad organizada más básica era el trokinche, lo cual correspondía a grupos

Dossiê Redes de Inter-aprendizaje: nuevas cartografías interculturales y algunas propuestas de transformación 
familiares, el crecimiento demográfico y la estructuración social, con cargos ya definidos obligaba a pasar a un nuevo tipo de organización que era el lof o lofche. Esta nueva unidad espacial territorialmente era más amplia que el trokinche y era donde se iba conformado lo que anteriormente se planteaba el tuwun y el kupalme con un rakizuam colectivo, o sea linajes familiares con funciones traspasadas de generación en generación y con un pensamiento o característica de ese tronco familiar.

Las características del lof se conocían a través del Az, o sea el cuerpo de normas y conocimiento específico que el grupo tenía sobre el territorio, relaciones sociales, económicas y políticas. Incluso la machi mantenía un conocimiento específico de los remedios del lugar, con lo cual cualquier cambio territorial alteraba este conocimiento.

Cuando aumentaba la población algunas personas de los grupos familiares ya compuestos emigraban para formar nuevos trokinche y volver a convertirse en lof mapu o lofche. Las relaciones familiares, políticas y económicas se seguían manteniendo y eso permitía formar un az mapu de mayor envergadura, ampliando los conocimientos del colectivo y los conocimientos específicos de las autoridades tradiciones, esto es lo que más adelante conformaba un rewe o ayjarewe. La integración a un rewe o un lof mapu por otras familias era un tema de conocimiento y de az mapu, recordamos lo que anteriormente planteábamos con los conceptos de akunche y amunche, como decíamos, la importancia de analizar las reglas de comportamiento de los que querían integrarse, pues se trataba de ver si se tenía el mismo Az, para lo que se realizaba lo que se conoce como weupitun, esta es una ceremonia que dura varios días y donde se ve si puede existir relación entre las costumbres que tienen los que están asentados en el lugar y los que provienen de afuera según lo que se decidía si se quedaban o no.

Esto también servía como un mecanismo de expansión territorial de ciertos linajes, los cuales eran poderosos por la extensión de sus rayñma (redes familiares), entendiendo que estas familias no sólo se extienden sobre el espacio físico, también lo hacen en otras dimensiones. Finalmente los grupos de alianzas familiares, políticas, económicas y militares dieron paso a los ayjarewe o quiñel mapu, donde se manejaba un az mapu, pasando ante distintas situaciones a formar un futal mapu, que era la unión de varios ayjarewe y que hacían futa trawun (grandes reuniones de carácter político y militar) y parlamentos (reuniones de carácter político con las autoridades chilenas).

Dossiê Redes de Inter-aprendizaje: nuevas cartografías interculturales y algunas propuestas de transformación R. Articul.const.saber, 2020, v.5: e65253 
Otro elemento para entender la utilización del espacio mapuche y el antagonismo en el uso del territorio por parte del chileno es lo que se relaciona con el ngen mapun y las fuerzas que existen como ngen (dueños de lugares al interior de un lof) que pueden ser menoko (sectores pantanosos) xayenco (esteros) winkul (cerros) mawida (montaña) o leufu (río). El az mapu obliga a respetar estos lugares y sus horarios, de lo contrario pueden ver perimotun (visiones), las cuales se presentan en distintas formas, enfermando o llegando a matar a la persona o animal que los vio. Es la machi quien puede salvarlo, siempre y cuando tenga el conocimiento específico del lugar y los remedios. Por ello se respetaban esos lugares y no se intervenían, porque esas fuerzas también ayudaban a que el mapuche tuviera una mejor vida. Por ejemplo, en la comunidad Curiche Epul en la comuna Nueva Imperial en la Provincia de Cautín las plantaciones de exótico de un fundo hizo que se fuera el ngen de xayenco y este comenzó a secarse (19-01-2003), este el mismo caso que Alfonso Reimán declara, en la comuna de Lumaco, Provincia de Malleco, y relacionado con la expansión forestal "Existe un rechazo absoluto en contra de la empresas forestales, porque mientras sigan existiendo... nosotros no vamos poder reconstruir nuestro territorio... Dentro de las plantaciones, hay lugares sagrados... En mi comunidad, antes habían lugares que hacían llover, pero ese Newen, esa fuerza que antes existía, ya no está" (ASOCIACIÓN ÑANCUCHEO, 26/04/2000).

Por lo tanto, el mapuche tiene una responsabilidad en su territorio, no sólo con el aspecto material de seguir viviendo y de asegurar algún nivel de desarrollo, sino también con la forma de organizarse socialmente y de vivir su espiritualidad, así como de construir y reconstruir sus espacios simbólicos. Es por ello que el actual discurso de identidades territoriales, al manifestar conceptos como control territorial, hace referencia a la necesidad de ejercer territorialidad, el cual es propio de los pueblos que alguna vez han ejercido soberanía, han poseído un territorio y lo han administrado, territorio del cual han surgido distintos lazos que van desde el asegurar la alimentación hasta la abstracción y creación de modelos sociales.

\section{La percepción del espacio mapuche desde los chilenos}

Para analizar el tema del espacio y la carga simbólica a las cuales son sometidos es necesario definir el concepto "espacio" como algo que "... no existe por sí solo, sino que se 
materializa, se concretiza en la existencia de algo real que le dé contenido el cual, socialmente, es consecuencia de la actuación de los agentes y actores sociales, sus interrelaciones y el tipo y forma de uso del territorio sobre el que se asienta" (GARCÍA JORDÁN, 1998, p. 230).

Esto quiere decir que son materializaciones y abstracciones a partir de la formación social y económica, como lo plantea Santos, ya que la acción de los agentes y sus instituciones son los que le dan forma al espacio y al territorio, puesto que lo social no está separado de lo ecológico (1996, p. 18). En este sentido, Santos (1999, p. 31) al igual que García Jordan (1998, p. 230) hacen referencia al territorio como la base para que se efectúe la construcción del espacio, por lo tanto, su estudio se justifica al analizar la construcción del espacio y sus usos o valor de uso, con lo cual se llega a la relación que existe entre el territorio y sus representaciones simbólicas. Para visualizar nuestro objetivo necesitamos mostrar en forma gráfica la percepción que los agentes manifiestan a través del discurso y sus representaciones simbólicas. Tenemos que tener presente que los agentes son componentes de una sociedad determinada o específica como lo es una formación social y económica y sus instituciones buscan plasmar en el territorio su visión de mundo o cosmovisión para dar forma a su sociedad y que se mantenga como unidad étnica en el tiempo. En el caso de Temuco puede ser catalogada como una Sociedad Fronteriza, articulada marginalmente a la economía y sociedad nacional, al igual que su proceso de globalización. Es una sociedad en la que se emiten discursos políticos e ideológicos con los cuales se plantea cuál debe ser el uso correcto del territorio, o sea, la cosmovisión fronteriza, en parte formada por la herencia cultural occidental y, por otro lado, por su carácter de frontera étnica, ante la cual la presencia y relación con el "otro" es explicada con categorías, por ejemplo mapuche = mal uso de la tierra, mapuche $=$ flojo, mapuche $=$ falta de educación. Como podemos ver, la diferencia es expresada incluso en un maniqueísmo en lo cual chileno $=$ civilizado, chileno $=$ buen uso del territorio, etc. Esto es lo que Barth plantea de la siguiente forma:

Los grupos étnicos en contacto "son categorías de adscripción e identificación" que organizan la interacción (BARTH, 1976, p.10-11). Para observar estos procesos de contacto cultural, "desviamos el foco de la investigación de la constitución interna y de la historia de los grupos étnicos para centrarlo en los límites étnicos y su persistencia" (loc.cit.). Distinguimos a un grupo étnico porque se autoperpetúa biológicamente, comparte unitariamente valores culturales, integra un espacio de comunicación e interacción y sus miembros se identifican a sí mismos

Dossiê Redes de Inter-aprendizaje: nuevas cartografías interculturales y algunas propuestas de transformación 
(nosotros) y son identificados por otros (ellos) constituyendo categorías diferenciables (loc.cit.) (GREBEG, 1997)

Tomando en cuenta lo planteado por Barth, nosotros, por nuestra parte, afirmamos que estas categorías dependen de la relación interétnica entre sociedades distintas. En el caso de que una sociedad quisiera dominar a otra las categorías comienzan a desarrollar una violencia extrema, llegando a la dicotomía que mostrábamos anteriormente.

Es bueno mencionar que esto no significa que toda la sociedad asuma un discurso antimapuche o antiindígena, siempre existen grupos disidentes. El tema es que para transformarse en agente debe poseer poder, ya sea de facto o por mecanismos que permitan la concentración de él. Es por ello que los agentes que generan estas imágenes se encuentran en la intelectualidad, dirigencia y grupos económicos los cuales las formulan bajo sus intereses y, de paso, correlacionan su pensamiento con los demás grupos de su sociedad, por eso la prensa y los medios de comunicación de masa se transforman en vehículos potentes para mantener una lógica, se hace un pacto con los grupos subalternos en el sentido de aceptar un proyecto en el cual ellos en cierta medida buscan reproducir o modificar en parte. A continuación analizaremos algunas citas aparecidas en medios de prensa y organismos que difunden la visión sobre el territorio mapuche y especialmente las cercanas a Temuco. Por ejemplo, este texto que influyó en gran parte de la intelectualidad de la frontera dice lo siguiente:

En la actualidad, numerosos grupos indígenas no sólo conservan lo que legítimamente les pertenece, como la lengua y sus atuendos típicos (la tierra la perdieron casi toda en el siglo pasado), sino lo que es más grave, conservan también, modos de vida, ceremoniales y técnicas de trabajo totalmente primitivas. Que la ruca siga siendo en muchos lugares el único y pintoresco "habitat" del medio indígena y que la tierra se siga trabajando tan absurdamente con los métodos más rudimentarios son quizás dos aspectos que componen el fiel retrato de la condición social y económica del indio.

Lo último es muy serio porque el mapuche se sustenta de la agricultura y de alguna crianza (ovejas) y porque las reducciones controlan todavía en la región alrededor de unas 400 mil hectáreas. Estas tierras se trabajan extensivamente en múltiples explotaciones y en propiedades sin cercar, en rotaciones inadecuadas, sin abonos y con sobrepastoreo de animales. Por preferencia al resultado de este tipo de explotación en las tierras situadas al sur de Temuco, se habla del cinturón suicida y la designación no es meramente imaginativa (CORFO, 1962, p. 372).

A esto se agrega el tema de la indivisión de las comunidades indígena (CORFO, 1962, p. 373), que se aprecia como un factor negativo por parte de esta institución, que promueven el

Dossiê Redes de Inter-aprendizaje: nuevas cartografías interculturales y algunas propuestas de transformación 
desarrollo de país y sobre todo vela por el interés de los "chilenos", quienes anunciaban con mayor ahínco eran los latifundistas, pero esto también era una oportunidad para los chilenos pobres que no poseían tierras como para vivir y desarrollarse. Al interior de este punto nos hemos encontrado que la percepción que manifiestan las autoridades y los grupos dominantes, tanto desde Santiago como Temuco, coincidieron en sus apreciaciones sobre el uso de los recursos de los mapuche, a continuación, podemos apreciar la interpretación de la elite local:

... quienes conocen la fisonomía de la producción agraria de Cautín saben muy bien que la barrera, el dique contra un mayor progreso de la economía regional, reside precisamente en las tierras que por estar en manos ociosas han venido a formar un circuito negativo, visible a una simple ojeada y conocido bajo la expresión gráfica de "el cinturón suicida de Cautín". Allí no florece el trabajo y el progreso, no filtra sus savias fecundas. Para estas tierras no rige, de manera alguna, el imperativo categórico de producir, que alcanza hoy ribetes de íntima urgencia y dramatismo, porque el hombre está padeciendo hambre en muchos rincones del orbe

...debieran los parlamentarios ir al estudio de un plan agrario espléndidamente organizado, ojalá a manera de cooperativas que tiendan a incrementar la producción de extensas zonas de lar agrícola sureño hasta hoy inexplotados por la característica de desidia indígena, por su falta de capitales y por su restringida visión de porvenir (Diario Austral, 1/07/1946).

Frente a esto, planteaban el hecho de que las ciudades de la IX región, en especial Temuco se encontraban ante un cinturón suicida. Foester \& Montecinos hacen un estudio sobre esta metáfora y datan este concepto desde la década de 1920 en el siglo pasado. En otros textos encontramos presente este concepto como en el Seminario de la provincia de Cautín realizado por la Universidad de Chile en su sede de Cautín en 1956, cabe destacar la ponencia del representante de la SOFO el cual va más lejos y acusa a la corporación Araucanía de llevar una campaña donde el mapuche se oponga a la subdivisión generando todo lo referente a la explicación de los cinturones suicidas:

Debemos encarar la solución del problema de la Comunidad Indígena y, al hacerlo, debemos rechazar los intereses políticos locales y del momento que siempre se han movido en torno a él y despojarnos de todo espíritu de sentimentalismo;... Si bien la solución del problema indígena tiene relación con la productividad agrícola de la zona, no olvidemos que la solución integral debe buscar la incorporación humana del indígena a la vida normal como asimismo de un estado civilizado (QUEZADA, 1956, p. 218).

Es necesario mencionar que entre las categorías que manejaba la SOFO en ese tiempo, se encontraban algunos conceptos como subcivilizado, que recientemente había abandonado la vida

Dossiê Redes de Inter-aprendizaje: nuevas cartografías interculturales y algunas propuestas de transformación

R. Articul.const.saber, 2020, v.5: e65253 
nómade y recién estaba desarrollando la explotación agrícola, de una organización política familiar inestable e incluso una incapacidad del indígena para entender el concepto de propiedad individual, lo que sí lo entendían los pueblos de mayor desarrollo cultural e institucional (op.cit; p.212). Queda claro que la visión sobre el otro, en el sentido como lo plantea Barth, era una visión negativa, se ve claramente la intención de mostrar una sociedad superior y otra inferior. En la cita textual que extrajimos, llama la atención que ante la amenaza que resultaban las comunidades mapuche era necesario apartarse de todo proindigenismo, porque la única vía era incorporar al indígena a la civilización, lo que significaba, en otras palabras, que dejara su cultura y se transformara en un chileno. Cabe recordar que en este contexto histórico, el objetivo final de los grupos dirigentes era la subdivisión de las comunidades mapuche, cabe señalar que a partir de 1927 la legislación toma este norte, pues el grupo de legislaciones anteriores busca, de alguna forma, proteger en cierta medida la propiedad colectiva mapuche, aunque ésta sea reduccionada. En este momento histórico también se hace necesario recordar que eran muy pocas las comunidades subdivididas y que las organizaciones políticas mapuche tenían como eje central evitar la subdivisión, ante lo cual se exacerbaron los ánimos, por ejemplo el mismo Quezada plantea en esta exposición lo siguiente: "El indígena tiene una enorme facilidad y gusto por los pleitos; parece que en ellos ha vaciado su espíritu guerrero de antaño y recurre al juzgado por cualquier nimiedad..." (op.cit; p. 216).

Es clara la negación y la forma de categorización del mapuche, sobre todo si se trata de defender sus tierras, pues se hace necesario recordar que en estos momentos los mapuches no estaban defendiendo su territorialidad, sino los pequeños grupos de tierra que el Estado chileno les había dejado. Con esto podemos recalcar que a medida que la sociedad mapuche luche por sus tierras o su territorialidad, como sucede en la actualidad, mayor será la virulencia de las categorías y el accionar de la sociedad dominante, incluso esto lo podemos afirmar en la actualidad, con el nuevo sistema de reforma procesal penal, en el cual se trata a los mapuche que intentan defender el territorio como terroristas,(de hecho, se les aplica la ley de seguridad interior del Estado) categorización a la cual se adhieren las transnacionales forestales y los latifundistas de la VII, IX y X región. Además del discurso de comunidades violentistas frente a las que mantienen el "dialogo".

Dossiê Redes de Inter-aprendizaje: nuevas cartografías interculturales y algunas propuestas de transformación R. Articul.const.saber, 2020, v.5: e65253 
Volviendo al caso de Foester \& Montecinos, obtienen el concepto de cinturón suicida en la prensa de la época, principalmente sobre los dichos de autoridades y latifundistas y como lo destacan hay un debate frente al concepto, en el cual, la parte ligada a los latifundistas y políticos relaciona a las comunidades con los campos mal aprovechados y que estos no serían capaces, aparentemente, de entregar todos los productos como hortalizas y de mases que aseguren la alimentación de la creciente población tanto de la provincia como sus ciudades. Por otro lado, el movimiento político mapuche, así como parte de la institucionalidad chilena, entre ellos parte de la iglesia católica, ven en esta aseveración una intención de apropiarse de los terrenos indígenas a través de la división de las comunidades. Es claro que los grupos chilenos también generan una propuesta para mejorar la producción agrícola que saca a los mapuche de sus costumbres productivas, convirtiéndolos en productores a la usanza chilena. Esto es sugerido por los participantes del seminario como por el estudio de la CORFO, con ello se niega a la sociedad mapuche y a su estructura económica, por lo que es imposible pensar en una relación horizontal entre mapuche y chilenos y sobre todo en tolerar las distintas formas de concebir el territorio.

Desde el enfoque de la geografía, relacionadas con la explicación de las relaciones espaciales entre distintos lugares, se podría pensar que los argumentos referidos estarían relacionados con la teoría de los lugares centrales, en la cual se encuentra una visión relativa a redes que permiten el surgimiento de los centros urbanos, la jerarquización entre éstos y los espacios rurales, a partir de las relaciones espaciales y actividades económicas, además se agrega la visión cuantitativa propuesta por Christaller y la acción planificadora, pese a que esta teoría tiene cierta circulación a finales de la década de 1930, siendo más difundido en la década del 60 en Inglaterra, parte de Europa y Estados Unidos, no podríamos precisar si esta postura influyó en el pensamiento de los intelectuales chilenos y parte de la elite dirigente de este país o si bien se relaciona con lo que Alejandro Saavedra intuyó sobre la teoría de raza inferior, esta teoría fundada a mediados del siglo XIX y basada en parte en el evolucionismo darwiniano, señalaba al mapuche en un estado inferior, incapaz de entender los conceptos "evolucionados" como propiedad privada, siendo uno de los representantes más radicales Benjamín Vicuña Mackenna "[el indio] no era sino un bruto indomable, enemigo de la civilización, porque sólo adora los vicios en que vive sumergido, la ociosidad, la embriaguez, la mentira, la traición y todo ese 
conjunto de abominaciones que constituyen la vida salvaje" (VICUÑA MACKENNA, 1939, pp, 406-407).

En esta ideología se sustentó la invasión al territorio mapuche, pero como lo señala Pinto (2000), existieron voces disidentes, las cuales fueron minoría frente a los intereses de la elite, pero para entender que esta apreciación es una lógica de pensamiento frente a la concepción territorial de corte occidental, podemos apreciar la siguiente ley:

Reglamento ley a favor de los indios dictado por la Junta de Gobierno de 1813, con acuerdo del Senado.

Deseando el Gobierno hacer efectivos los ardientes conatos con que proclama fraternidad, igualdad y prosperidad de los indios y, teniendo una constante experiencia de la extrema miseria, inercia, incivilidad, la falta de moral y educación en que viven abandonados en los campos, con el supuesto nombre de pueblos, y que, a pesar de las providencias que hasta ahora se han tomado y (tal vez por ella misma) se aumenta la degradación y vicios, que también quedaría condenada su posteridad, que debe ser el ornamento de la patria (JARA, 1956, p. 25).

Esta ley fue diseñada para terminar con la pequeña propiedad indígena que quedaba al norte del Biobio, podríamos decir que es el exterminio de la población mapuche pikunche y su propiedad. En los artículos siguientes se presentan en forma gráfica las intenciones y el modo de desestructurar la forma de vida mapuche. Cuando hablamos que esto es una lógica, que supera a un hecho histórico de corta duración y a una ideología, lo planteamos, en que es parte de una cosmovisión, de entender en forma paradigmática el uso de los elementos que acompañan al hombre, es parte de un hecho cultural como lo señala Javier San Martín (1997) en el sentido de concebirlo como una forma de utilización universal o étnico, por lo cual existe una continuidad en una sociedad, con sus construcciones de mundo. Para el caso chileno, el uso del territorio esta marcado por una verticalidad entre el hombre y la naturaleza, es por ello que se la caracteriza como un recurso productivo y de ahí su valor de uso y valor de cambio. El territorio pasa a ser un bien intercambiable.

La percepción de los grupos intelectuales y dirigentes, sobre todo en una relación de colonialismo interno, donde se concentra el poder, se fijan las políticas a los sectores marginado desde una verticalidad, provocan imágenes sobre grupos subalternos como las comunidades, que han sido estigmatizados, se han creado incluso legislaciones para destruir a las comunidades , como se puede ver la lógica en el sentido como la presenta Henry Lefevre de orden, obvia el

Dossiê Redes de Inter-aprendizaje: nuevas cartografías interculturales y algunas propuestas de transformación 
significado que posee el mapuche sobre su territorio, la lógica de Homo Economicus de Marx pareciera ser más fuerte, pues todo espacio debe ser productivo. El interés intelectual es volver a las comunidades productivas cuestión que desde 1960 hasta el día de hoy se discute, obviamente el interés productivo debe tener una lógica, un orden territorial, el occidental. Sin duda las contradicciones se han manifestado cada vez más fuerte en el sentido que la sociedad mapuche hoy reivindica control territorial y la sociedad chilena ha insistido tozudamente en cifrar su desarrollo a partir del territorio mapuche, emblemáticos son los casos de Ralko, By Pass en Temuco, crecimiento inmobiliario de Temuco, ampliación territorial de las forestales y vertederos en territorio mapuche y como lo ha planteado Ricardo Lagos donde no se puede sacrificar el desarrollo de los chilenos por una minoría, minoría que en este caso seríamos los mapuche.

\subsection{Del antagonismo al acercamiento}

A continuación planearemos una serie de conceptos utilizados por geógrafos o intelectuales dedicados al tema étnico y espacial, bajo un análisis crítico referido a cómo las ciencias sociales podrían servir de puente entre las reividincaciones sociales y políticas de los pueblos originarios.

Creemos que uno de los conceptos más sobreutilizados, o del cual se ha abusado en la ciencias sociales, es el de etno, el cual, por lo general, ha sido utilizado para delimitar el objeto de estudio, en el fondo ha actuado como una categoría operacional, la cual ha permitido establecer categorizaciones que sólo han servido para, de alguna forma, mantener un concepto globalizador, así como el concepto indígena, con el fin de ocultar la subordinación de la cual somos parte los pueblos originarios; por ejemplo, el concepto de etnogeografía, que propone Horacio Larraín, en su texto del mismo nombre y que pertenece a la colección Geografía de Chile del Instituto Geográfico Militar, donde a través de todo el capítulo dos va relacionando las particularidades de los objetos de estudios de otras ciencias sociales, en especial las que se han desprendido de la antropología, como son la antropología social y cultural, al mismo tiempo, que la arqueología y la etnohistoria, en la cual sus objetos coinciden en trabajar con grupos étnicos y en distintos tiempos. Los elementos reflejados en el esquema que nos presenta Larrain, son los mismos utilizados en la geografía, basados en explicar las estructuras sociales a partir las estructuras físicas, sociales y culturales, pero obviando las dinámicas de las sociedades en cuanto a su poder

Dossiê Redes de Inter-aprendizaje: nuevas cartografías interculturales y algunas propuestas de transformación

R. Articul.const.saber, 2020, v.5: e65253 
de transformación. Esta crítica es parecida a la que Santos señalaba sobre la geografía al decir “... que la Geografía se interesó más por la forma de las cosas que por su formación. Su dominio no era el de las dinámicas sociales que crean y transforman las formas, sino el de las cosas ya cristalizadas ..." (SANTOS, 1996, p.17)

En cuanto a su propuesta, en el fondo, es un estudio de la comunidad humana (etno) (LARRARÍN; 1986), sobre todo basado en el paisaje, entendiendo éste como un concepto que encierra toda la característica de una cristalización. Para Larrain, lo etno no involucra la epistemología mapuche de cómo considera y ordena su espacio, es más, su estudio opera con las categorías y percepciones de los cronistas del siglo XVI, lo cual lleva a reproducir la percepción espacial de los hispanos y a ver la forma en cómo construían cognitivamente el espacio para ocuparlo. Creemos necesario, para ello, ampliar el concepto etno, de forma de entender la lógica del otro, al utilizar y ordenar su espacio. En ello se encuentra la epistemología del sujeto a estudiar sumada a la epistemología del sujeto investigador, de la cual, a partir de las diferencias, contradicciones y antagonismos se pueden establecer los consensos concretos, en los pactos que superen la subordinación y establezcan nuevas formas de relacionarse. Como apreciamos en los dos casos anteriores sobre percepción territorial, ambas dependen de reconocerse y de los antagonismos para buscar fórmulas políticas que permitan cerrar el ciclo de colonialismo ante los estados nacionales y establecer un dialogo sustentado en el reconocimiento de ambas territorialidades.

Ampliando el concepto etno, también surgen otras categorías espaciales como pueden ser los conceptos de etno-territorios y tierras indígenas, ambos encierran un trasfondo político, en el cual ambos no sólo responden a criterios de investigación, sino que están ligados al tema de reividincación de los pueblos originaros, los cuales, por ser colectivos, son sujetos a derechos políticos, expresados en legislaciones que los estados nacionales crean o ratifican en acuerdos internacionales, como, por ejemplo, la discusión en Chile para ratificar el convenio 169 de la OIT. Es por ello que Bengoa señala que al hablar de tierras indígenas en un momento de la historia, todas fueron tierras indígenas y, por tanto, hay que buscar un concepto operacional que sirva a una legislación de reconocimiento de tierras (1992, p. 41). En este sentido volvemos a lo planteado en la primera parte de esta ponencia, el investigador no existe fuera de su sociedad, sino influida en ella, desarrolla sus conceptos y enfoques.

Dossiê Redes de Inter-aprendizaje: nuevas cartografías interculturales y algunas propuestas de transformación R. Articul.const.saber, 2020, v.5: e65253 
Para Raúl Molina, quien utiliza el concepto etno para individualizar el territorio desde la perspectiva de quien utiliza o quien reivindica un espacio, señala que

Los etno-territorios constituyen una categoría que da cuenta de los espacios habitados
por pueblos indígenas o una parte de éstos, que posee por características, encontrarse
delimitados por hitos geográficos, reconocidos socialmente por una o más agrupaciones
de una misma etnia o de otra distinta. Estos territorios son valorizados por los indígenas,
al asignarle un contenido político, económico, social y religioso (MOLINA, 1995, p.
111).

Bajo esta perspectiva y, explicada por el autor en mayor profundidad en su artículo, lleva a pensar que frente a las relaciones interétnicas entre sociedades distintas, sobre todo por el elemento Estado, se hace necesario distinguir o agrupar estas categorías de forma distintas, pero reconociendo en ellas que existe una dinámica, una forma de ejercer territorialidad, sin duda esta categoría actúa como una frontera étnica más que territorial. Esto es muy parecido a lo que plantea Domique Temple al referirse al uso de territorio, en el cual el uso de la tierra bajo la visión indígena sería su territorialidad (1989, p. 148), el transgredir la propiedad indígena significa vulnerarlo y despojarlo de su proyecto como sociedad. Esta territorialidad, basada en los elementos étnicos con las fronteras étnicas planteadas por Barth, es lo que Claval llama territorialidad cerrada diferenciada a la que poseen los estados nacionales, a la cual define como territorialidad abierta (1987, p.239), sin duda los conceptos recién mencionados pueden generar cierta reticencia en el sentido que pueden agudizar las contradicciones actuales, lo que a nuestro juicio, es la posibilidad de llevar a cerrar el ciclo y comenzar uno nuevo.

Para analizar los territorios de los pueblos originarios hay que analizar desde una doble perspectiva, como intentamos señalarlos anteriormente, uno es desde los discursos que generan la diferenciación étnica y por otro, bajo las formas culturales, pero en ambas se encuentra un elemento transversal que es el poder, lo que está presente en toda acción de los individuos no concordamos con lo planteado por Barth en que:

el foco de la investigación es el límite étnico que define al grupo y no el contenido cultural que encierra. Por su puesto, los límites a los cuales debemos dedicar nuestra atención son límites sociales, aunque bien puedan contar con su concomitante territorial... Los grupos étnicos no están basados simplemente o necesariamente en la ocupación de territorios exclusivos; necesitamos analizar los diferentes medios por los cuales logran conservarse, pues no es sólo mediante un reclutamiento definitivo, sino en virtud de una expresión y una ratificación continuas (BARTH, 1976, p. 17).

Dossiê Redes de Inter-aprendizaje: nuevas cartografías interculturales y algunas propuestas de transformación 
Puesto que de los usos, de los contenidos culturales, es de donde proviene la diferenciación, no es solo un discurso o una ideología indígena, sino que es la forma en que las sociedades buscan mantenerse, obviamente no en un sistema cerrado, es por ello que generan la diferenciación, para poder incorporar lo del otro, pero su objetivo es su cultura, es por ello que para desarrollarse necesita un territorio, pues en el uso se encuentra la diferencia de forma concreta. Pero al mismo modo se generan las interconexiones entre etnias, esto es lo que ha permitido hablar de relaciones fronterizas o relaciones interétnicas, ambas escuelas fundadas por distintas disciplinas, la historia y la antropología, generan discursos de cómo fueron estos procesos de interconexiones. Este concepto desarrollado por Wolf en la introducción de su libro Europa y la gente sin Historia nos plantea de cómo las culturas históricamente se han interconectados a partir intercambios culturales, sean en formas verticales u horizontales, son las culturas que desarrollan estos procesos las que se mantienen en el tiempo.

En este último punto la Geografía Cultural, desarrollada por Claval, nos lleva a estudiar los elementos que relacionan al hombre con su medioambiente, en sus formas de herencia cultural y comunicación, en cuanto a su construcción como sociedad, en su discurso, en la diferenciación social, en la impresión o huella, que como sociedad, dejan en el espacio y en la interpretación de este (CLAVAL, 1999, p. 17-19). Pero volvemos a recordar que para nosotros el punto intermedio es el analizar a las sociedades en su contacto, pero también en sus culturas y en los elementos que la hacen propias.

Es por ello que es necesario estudiar al espacio en sus formas y sus formaciones, en cuanto a que son las sociedades las que le dan o le imprimen su sello particular, pero en formas de interconexiones, como lo señala Santos “... es el espacio de todas las empresas, de todas las instituciones, de todas las personas, de todas; ... Porque la existencia no excluye la presencia de la sociedad, el trabajo de cada persona contribuye a la producción de ese cotidiano que, cada vez es parte de nuestro trabajo..." (SANTOS, 1999, p. 32)

Y con ello recalcamos que si queremos que los conflictos que poseen los pueblos originarios con los estados nacionales se resuelvan, no podemos pensar en espacios homogéneos o en sus particulares extraídas de sus formas. En este sentido es un concepto de espacio y región hay que verlo desde una posición holística de análisis, que involucra a toda la sociedad o 
sociedades y el medioambiente, en este sentido implica la necesidad de involucrar la dimensión territorial a la institucionalidad, ejercicio que siempre se hizo solo sectorizado a la institución a fin, como la localización de la industria o el sistema educativo, pero sin involucrar el flujo que tienen las distintas instituciones sobre ellas y el espacio a partir de un territorio. Sin duda como los plantea Santos el territorio es un agregado, lo que interesa a la sociedad y al investigador es su uso, en como este es parte de una sociedad determinada (31), planteando el territorio como parte de una sociedad o una etnia, como lo manifiesta Breton:

\begin{abstract}
Después de la lengua, de la demografía y del origen antropológico, el territorio es el cuarto elemento que permite delimitar a la etnia, el cuarto elemento en el que se fundamenta la estructura étnica. En cuanto a la sociedad global, completa, autosuficiente, la etnia ocupa en la superficie terrestre una porción que le es propia. Es el marco físico donde la etnia se ha fijado, e incluso muchas veces donde se ha constituido a partir de elementos de procedencia diversa. La etnia ha adoptado a este medio natural y lo ha transformado en menor o mayor grado para la utilización de los recursos. Cuanto más desarrollada, sofisticada y exigente es la economía, más modifica el marco ecológico, más <<humaniza〉> el paisaje. Cuanto más densa es la ocupación del territorio más profundas e irreversibles son estas transformaciones (BRETON, 1983, p.46).
\end{abstract}

Es a partir de esta base que la lucha por el control territorial o ejercer la territorialidad se vuelve vital, es uno de los elementos que permite avanzar el colectivo. En esta lógica se establece que "El territorio de cada etnia varía en extensión, a su medida y a su manera: ..." (p. 48), lo cual nos indica que no hay un determinismo geográfico en la ocupación y relación con el medio natural es más "En ocasiones el espacio es común a varias etnias, las cuales hacen uso distinto de las partes del terreno en función de sus respectivos géneros de vida y de los desplazamientos temporeros de unos y de otros...” (p. 49). Esto es un elemento sumamente importante ya que para nuestra reconstrucción territorial debemos partir de la visión que actúan dos grupos étnicos distintos los chilenos y los mapuche, estableciéndose que uno de los grupos está destruyendo la territorialidad del otro, le están estrangulando su posibilidad de desarrollo. Estudios relacionados con la utilización de los recursos naturales o relacionados con el tema de la propiedad, pues es este último concepto valido para el reconocimiento frente al Estado como lo señala Bengoa (1992, p. 41) o es lo que explica Molina \& Correa al plantear que existen dos formas de analizar dos formas del territorio, respecto al tema de la propiedad indígena, uno es la historia legal, la cual es la reconocida a través de las instituciones del Estado y la otra es la de los propios mapuche (1996: 207), en ella, como hemos planteado, se trata del tema de la territorialidad, no es

Dossiê Redes de Inter-aprendizaje: nuevas cartografías interculturales y algunas propuestas de transformación

R. Articul.const.saber, 2020, v.5: e65253 
raro ver cómo las propias autoridades mapuche o los estudios de etnobotánico o de expansión forestal han señalado que la destrucción no sólo afecta al territorio, sino a la sociedad mapuche. Lo más grave es que actualmente los pueblos originarios no cuentan con instrumentos legales para enfrentar con éxito estos problemas y, por otro lado, no existe por parte de la clase política y la elite chilena gestos para cambiar esta situación. Los mapuche siguen recordando cómo sus antiguos gobernaban el territorio antes de la llegada del chileno, existen los testimonios escritos y los instrumentos legales que prueban que eso fue así, pero falta la voluntad, por parte de los gobiernos y parte de la sociedad chilena, para establecer una nueva lógica de entendimiento y de uso del territorio sobre todo en las regiones, donde el diálogo se transforma en asistencialismo y un falso de consenso respecto al problema indígena.

Sin duda los desafíos que aquí planteamos nos deben llevar a una nueva forma de entender el espacio, diferente a como por lo general se ha entendido y se ha enseñado. Hoy en día los desafíos están en cómo vivir el espacio, tener que generar nuevos tipos de sentimientos, en el fondo, aprender a crear un lugar donde mapuche y chilenos convivan con una visión de desarrollo que no resulte antagónica, o sea que, fundamentalmente, pasa por crear un lugar como lo señala Paulsem “... esa porción de espacio en donde se produce la simbiosis de los sentimientos personales con lo simbólico y lo colectivo. El lugar no es una colección de eventos y objetos observables, sino que es más bien el receptáculo de significados” (2001, p. 210).

En otras palabras se relaciona con lo que anteriormente planteaba Santos, en superar el análisis de locacional y sobre todo aislado de los sujetos que construyen dichas formas. Esto nos lleva a la necesidad de flexibilizar nuestras verdades, establecer consensos sobre el uso de los recursos, por lo que es necesario retomar lo que Graciela Uribe planteaba en lo que se refería a la Geografía Política que, en el fondo, es reconocer el fenómeno del poder, al igual que el de los sentimientos, para reestablecer las relaciones espaciales y sociales. Por otra parte, se hace necesario una visión más fenomenológica que cuestione la forma cómo estructuramos el conocimiento, en admitir que el mundo se construye en interrelaciones y el sujeto que las estudia no se puede desligar de ella.

Por la necesidad de reorientar los textos que tienen que ver con la enseñanza de la historia y del espacio, que en las regiones o territorios con problemas de antagonismos espaciales sean los encargados de producir su material, creando para ello instancias de investigación y de 
participación política, que tengan la oportunidad de conocer la realidad de su región a partir de las relaciones entre los sujetos. Se hace necesario que los sujetos sociales sancionen si lo que se ha investigado corresponde a su realidad o a un imaginario.

\section{A Modo de Conclusión}

Como podemos apreciar no existe un mundo y una metodología para conocerlo, esto queda en evidencia cuando queremos explicar las relaciones interétnicas a partir de los usos del territorio y vemos que se mantienen dos sociedades distintas, aunque para el caso mapuche, en subordinación a otra. Los conflictos territoriales se han acrecentado por la falta de voluntad y de enfoques que permitan entender que la pobreza, la marginalidad del pueblo mapuche y su discurso reivindicativo parte desde la necesidad de mantener un colectivo, pero relacionado a otras sociedades. La ciencia no ha sabido responder a las necesidades y realidades sociales de los otros, simplemente porque se han quedado en su condición de objetos de estudios, su interés ha estado en presentar una explicación coherente o racional a la forma de pensamiento occidental. La posibilidad de conocer la realidad mapuche pasa por establecer nuevas instancias políticas para formalizar consensos respecto a la investigación y las posibilidades de desarrollo que ocupan dos sociedades en un mismo territorio.

El kimun mapuche aparece como una alternativa para generar nuevos tipos de conocimientos, de acercamientos y diálogos, permite reconocer y diferenciar al otro, pero al mismo tiempo, asienta la plataforma para establecer los acuerdos, los elementos mínimos para que el pueblo mapuche se pueda desarrollar. Los conceptos como territorialidad o control territorial mapuche se fundamentan en el kimun mapuche, por lo que no es un discurso que elaboran unos pocos, sino la construcción de miles de generaciones mapuche, con una estructura abierta que le permite enfrentar los actuales desafíos de la globalización.

La labor del intelectual, tanto del que investiga como del que enseña, es establecer un sistema más abierto en cuanto a perspectivas de conocimiento, pero al mismo tiempo reconocer que su opinión no sólo es intelectual, también es política, es por ello que debe explicitar el contexto bajo el cual observa al otro, describir cómo se interrelaciona con su supuesto objeto de estudio, plantear qué tipo de realidad está generando y cuáles serían las consecuencias de esto. La

Dossiê Redes de Inter-aprendizaje: nuevas cartografías interculturales y algunas propuestas de transformación 
dinámica del poder no es ajena a la ciencia y la enseñanza, se vive día a día, es cotidiana, al mismo tiempo que las emociones, es por ello que entender cómo se producen los sentimientos, que elementos juegan en la construcción social de los territorios y admitir la adscripción al espacio que uno desearía crear o desarrollar, pero en un marco de flexibilidad y tolerancia que nos lleve a modificar las redes de poder actuales.

Para los mapuche los desafíos de la territorialidad no se terminan en la comunidad, sino que empiezan ahí, en la ruralidad mapuche y deben viajar, posesionarse de lo urbano. El mapuche actual, mayoritariamente urbano, debe reclutarse en las comunidades, empaparse de su lofche, para apropiarse de la ciudad, no quedarse sumergido en el anonimato, expresar su territorialidad a partir de la modificación del espacio urbano, convertirse en colectivo, esto quiere decir, reforzar las redes al interior de la ciudad como con los lofche. Es por ello que en su momento necesitará construir institucionalidad propia, instituciones que le permitan desarrollarse como colectivo, sean estos centros de diversión, centros médicos, espacios rituales, etc, elementos que lo agrupen, pero que no discriminen al otro, al contrario integren al otro a nuestra cultura, para ello se necesitan nuevos pactos e investigaciones. Estos son los desafíos que, de algún modo, nos van a permitir desarrollarnos como sociedades distintas, pero sin perjudicar al otro. Solo la madurez política de los pueblos permitirá la justicia social, un orden social humano y la convivencia pluriétnica.

\section{Referencias}

ACTAS Primer y Tercer Encuentro Nacional de Salud de Pueblos Indígenas; Chile 1996 y 1999.

ACTA Pueblo Mapuche, Medio Ambiente y Organizaciones No Gubernamentales (ONGs); Chile, 1991.

AYLWIN, José (comp.) Políticas Públicas y Pueblo Mapuche; Instituto de Estudios Indígenas UFRO \& Ediciones ESCAPARATE; Temuco-Chile, 2001.

AUKIÑ Wallmapu Ngulam El Pueblo Mapuche y sus Derechos Fundamentales; Chile, 1997

BARTH, Fredrik (comp.) Los grupos étnicos y sus fronteras. La organización social de las diferencias culturales. Fondo de Cultura Económica. México, 1976.

BOCCARA, Guillaume \& Galindo, Silvia (Ed) Lógica Mestiza en América. Instituto de Estudios Indígena, Universidad de la Frontera; Temuco-Chile, 2000. 
BOCCARA, Guillaume. (a) Análisis de un proceso de etnogénesis: el caso reche-mapuche de Chile en la época colonial. Memoria Americana. España, 1998.

-(b) El poder creador; Tipos de poder y estrategias de sujeción en la Frontera sur de Chile en la época colonial. Anuario de Estudios Hispano-Américanos. Sevilla, España, 1999.

BOSQUE, Joaquín; Ortega, Francisco Comentarios de textos geográficos (Historia y crítica del pensamiento geográfico); Oikos-Taus ediciones; España, 1995.

BRAGG, Catherine \& Hawenstein, Enrique \& Latsague, Mirtha Transecto Etnobótanico del Sector Mapuche; en Rev. Cultura-Hombre-Sociedad; Volumen 3 No 2; Revista de Ciencias sociales y Humanas, Universidad Católica de Temuco; Chile, 1992.

BRETÓN, Ronald Las Etnias. Oikos-tau ediciones. España, 1983.

BONFIL BATALLA, Guillermo. La teoría del control cultural en el estudio de procesos étnicos. Sin fecha de edición.

BOURDIEU, Pierre. (a) Capital cultural, escuela y espacio social. Siglo XXI edit.; $2^{\text {a }}$ edición. México, 1998.

(b) Intelectuales, Política y Poder; EUDEBA; primera reimpresión; Argentina, 2000.

CANIULLAN, Víctor \& Quidel, José Las identidades Territoriales. Bases y Fundamentos desde una perspectiva de Conocimiento mapunche; Documento elaborado para los talleres de discusión interna de la COTAM, 2002.

CANIUQUEO, S. Antagonismo en las Percepciones Territoriales, Un Marco de Interpretación. Revista Historia y Geografía, N 19, 9-48, 2005.

CARO PUENTES, Araceli; Duran Pérez, Teresa \& Tereucan Angulo, Julio (editores) Estilos desarrollos en América Latina. Identidad - cultura -Territorio -Medio Ambiente. Un aporte para la discusión. Universidad de la Frontera. Temuco, Chile, 1999.

CLAVAL, Paul; (a) Geografía Humana y economía contemporánea; Oikos-Tau Ediciones; España, 1987.

(b) Geografía Cultural. Editorial Universitaria de Buenos Aires. Argentina, 1999.

COMISIÓN CHILENA DE DERECHOS HUMANOS Pueblo, tierra, Desarrollo: Conceptos fundamentales para una nueva ley indígena; Centro Internacional para los derechos humanos y desarrollo Democrático; Chile, 1992.

COÑA Y MOESBACH Lonko Pascual Caña ñi tuculpazungun. Testimonio de un cacique mapuche; quinta edición; Pehuen ediciones; Chile, 1995.

CORREA, Martín \& Molina, Raúl Territorio y Comunidades Pehuenches del Alto Bío Bío; CONADI; Santiago-Chile, 1996. 
FUTATRAWUN KINEWAN MAPUCHE COORDINADORA UNITARIA MAPUCHE Declaración Pública. En revista Nutram Año IV- N³ - 1988, Santiago, Chile, 1988.

HARVEY, David. Teoría, Leyes y modelos en geografía; Alianza Universidad; España, 1969.

HARRIS, Marvin. Jefes, Cabecillas y Abusones; Alianza Editorial; España, 1993.

JARA, Álvaro. Legislación Indigenista de Chile; Ediciones Especiales de Instituto Indigenista Interamericano; México, 1956.

LARRAÍN, Horacio. Etnogeografía; Geografía de Chile, tomo XVI. Instituto Geográfico Militar. Chile, 1987.

LEEF, Enrique. Ecología y Capital. Racionalidad ambiental, democracia partiocipativa y desarrollo sustentable; Siglo XXI edit.; segunda edición; México, 1994.

LIWEN; La Corporación Araucana: (1938-1969) Una aproximación desde la historia oral mapuche; Centros de Estudios y Documentación Mapuche; Borrador; por publicar.

MCFALL, Sara (comp.) Territorio Mapuche y Expansión Forestal; Instituto de Estudios Indígenas UFRO \& Ediciones ESCAPARATE; Temuco-Chile, 2001.

MARIMÁN, Pablo (comp.) Parlamento y Territorio Mapuche; Instituto de Estudios Indígenas UFRO \& Ediciones ESCAPARATE; Temuco-Chile, 2002.

MAÑKELEF, Manuel \&Guevara, Tomás Kiñe mufü trokiñche ñi piel. Historias de familias/ siglo XIX; CEDEM Liwen \& CoLibris Ediciones; Temuco, Santiago Chile, 2002.

MOLINA, Raúl. Reconstrucción de los Etno-territorios; Institutos de Estudios Indígenas UFRO; Temuco-Chile, 1995.

MORALES, Roberto (comp.) Municipios y Participación (o Exclusión) Mapuche, Instituto de Estudios Indígenas UFRO \& Ediciones ESCAPARATE; Temuco-Chile, 2001.

MORALES, Roberto (com.). Territorialidad Mapuche en el Siglo XX, Instituto de Estudios Indígenas UFRO \& Ediciones ESCAPARATE; Temuco-Chile, 2002.

NACIONES UNIDAS DERECHOS HUMANOS. Los derechos de los Pueblos Indígenas; Folleto informativo No 9; EEU, 1992.

ORTEGA, José (2000) Los horizontes de la geografía. Teoría de la geografía; Edit. Ariel, S.A.; España.

PAULSEN, Abraham. Lugar, Acción: El Enfoque de la Geografía Humanística; en Boletín Historia y Geografía No 15; Universidad Católica Cardenal Raúl Silva Henríquez; SantiagoChile, 2001. 
PINTO, Jorge. De la inclusión a la exclusión. La formación del estado, la nación y el pueblo mapuche; primera edición; Instituto de Estudios Avanzado (IDEA); Universidad de Santiago de Chile; Santiago, 2000.

PINTO Rodriguez, Jorge (2003) La Formación del Estado y la Nación, y El Pueblo Mapuche. De La Inclusión A La Exclusión. $2^{\mathrm{a}}$ edición. Ediciones de la Direcci6n de Bibliotecas, Archivos y Museos, Centro de Investigaciones Diego Barros Arana. Santiago Chile.

QUEZADA Cid, Carlos. Legislación Indígena, representante SOFO panel; "Geografía Humana. El indígena en la Provincia"; en "Seminario de Investigación sobre el Desarrollo de la Provincia de Cautín"; Ediciones del departamento de Extensión Cultural de la Universidad de Chile; Temuco-Chile, 1956.

QUIDEL, José \& Jineo, Fernando. Las raíces para nuestro cultivo; en Caro, Aracely \& Duran, Teresa \& Tereucan, Julio (edit.); "Estilos desarrollos en América Latina. Identidad-CulturaTerritorio-Medio Ambiente. Un aporte para la discusión"; Universidad de la Frontera; TemucoChile, 1999.

RUIZ ALDEA, Pedro. Los Araucanos y sus Costumbres; Tercera Edición; Ediciones y la Ciudad, Municipalidad de Concepción. Chile, 1999.

RUIZ, Enrique. Las entrañas del Descontento. Cerco de Las forestales estrangula a familias indígenas. Comunidades Mapuche en conflicto; en Sin Censura. Reportajes ganadores, "Concurso Periodismo Joven"; Ediciones LOM; Santiago-Chile, 2000.

SAN MARTÍN, Javier. Fenomenología y Antropología; Edit. Almagesto; Argentina, 1997.

SANTOS, Milton. (a) De la Totalidad al Lugar; Oikos-Tau Ediciones; España.

(b) (1999). El territorio: un agregado de espacios banales. En Miguel Panadero y Francisco Cebrián coordinadores. América Latina; lógica locales, lógicas globales. Ediciones de la Universidad de Castilla-La Mancha. Cuenca, España, 1996.

TEMPLE, Dominique. Estructura Comunitaria y Reciprocidad del quid-pro-quo histórico al economicidio; hisbol-CHITAKOLLA; la Paz-Bolivia, 1989.

URIBE ORTEGA, Gabriela. Geografía y Sociedad. Exploraciones en compromisos y propuestas actuales. Centro de Investigaciones Científicas. México, 1998.

Submetido em 25 de agosto de 2020.

Aceito em 05 de outubro de 2020.

Publicado em 16 de outubro de 2020.

Dossiê Redes de Inter-aprendizaje: nuevas cartografías interculturales y algunas propuestas de transformación

R. Articul.const.saber, 2020, v.5: e65253 